\title{
Vitamin D protects dopaminergic neurons against neuroinflammation and oxidative stress in hemiparkinsonian rats
}

\author{
Ludmila A R Lima', Maria Janice P Lopes ${ }^{2}$, Roberta O Costa², Francisco Arnaldo V Lima ${ }^{1}$, Kelly Rose T Neves ${ }^{1}$, \\ lana B F Calou ${ }^{3}$, Geanne M Andrade ${ }^{1}$ and Glauce S B Viana ${ }^{1 *}$
}

\begin{abstract}
Background: The deficiency in 1a, 25-dihydroxyvitamin D3 (VD3) seems to increase the risk for neurodegenerative pathologies, including Parkinson's disease (PD). The majority of its actions are mediated by the transcription factor, VD3 receptor (VD3R).

Methods: The neuroprotective effects of VD3 were investigated on a PD model. Male Wistar rats were divided into the following groups: sham-operated (SO), 6-OHDA-lesioned (non-treated), and 6-OHDA-lesioned and treated with VD3 (7 days before the lesion, pre-treatment or for 14 days after the 6-OHDA striatal lesion, post-treatment). Afterwards, the animals were subjected to behavioral tests and euthanized for striatal neurochemical and immunohistochemical assays. The data were analyzed by ANOVA and the Tukey test and considered significant for $p<0.05$.

Results: We showed that pre- or post-treatments with VD3 reversed behavioral changes and improved the decreased DA contents of the 6-OHDA group. In addition, VD3 reduced the oxidative stress, increased (TH and DAT), and reduced (TNF-alpha) immunostainings in the lesioned striata. While significant decreases in VD3R immunoreactivity were observed after the 6-OHDA lesion, these changes were blocked after VD3 preor post-treatments. We showed that VD3 offers neuroprotection, decreasing behavioral changes, DA depletion, and oxidative stress. In addition, it reverses partially or completely TH, DAT, TNF-alpha, and VD3R decreases of immunoreactivities in the non-treated 6-OHDA group.
\end{abstract}

Conclusions: Taken together, VD3 effects could result from its anti-inflammatory and antioxidant actions and from its actions on VD3R. These findings should stimulate translational research towards the VD3 potential for prevention or treatment of neurodegenerative diseases, as PD.

Keywords: Parkinson's disease and neurodegeneration, Vitamin D, Oxidative stress, Neuroinflammation, Vitamin $D$ receptors

\section{Background}

Parkinson's disease (PD) is a consequence of dopaminergic neurodegeneration in the substantia nigra pars compacta $(S N p c)$. The cardinal signs of the disease are bradykinesia, muscle rigidity, rest tremor, and postural instability. The causes of the beginning of neuronal death are still unclear. Nevertheless, the already known misfolded proteins, specifically $\alpha$-synuclein, and the mitochondrial dysfunction

\footnotetext{
* Correspondence: gbviana@live.com

${ }^{1}$ Faculty of Medicine, Federal University of Ceará (UFC), Rua Barbosa de Freitas, 130/1100, Fortaleza, CE 60170-020, Brazil

Full list of author information is available at the end of the article
}

observed in PD patients create a favorable environment to oxidative stress and neuroinflammation, nurturing a cascade of events that amplify the death signal [1]. The major approaches to PD therapy remain to be the restoration of dopaminergic transmission in basal ganglia, since clinically effective neuroprotective agents that may be able to slow the degeneration of nigral dopaminergic neurons, have not been identified yet [2].

There is therefore a critical need for new drugs and drug targets focusing on the protection of dopaminergic neurons from degeneration [3]. Thus, the choice of a single target against the complex and poorly understood

(C) The Author(s). 2018 Open Access This article is distributed under the terms of the Creative Commons Attribution 4.0 International License (http://creativecommons.org/licenses/by/4.0/), which permits unrestricted use, distribution, and 
pathophysiology of PD has been shown inefficient [4], what guides the search for new drugs with pleotropic properties, as vitamin D (VD3). Appropriate levels of VD3, whose active metabolite is $1 \alpha, 25$-dihydroxy-vitamin D or simply calcitriol, are essential for the normal neuronal function, such as immunomodulation and detoxification processes [5]. VD3 shows a potent antioxidant activity, being able to reduce lipid peroxidation and to improve brain enzyme activity. A decline in VD3 levels will lead to a dysregulation in $\mathrm{ROS}$ and $\mathrm{Ca}^{2+}$ signaling pathways and, in some neurodegenerative pathologies as Alzheimer's disease (AD), it might initiate beta-amyloid formation which progresses to neuronal death and dementia $[6,7]$. Some clinical studies point out to the insufficient levels of vitamin D in patients with PD, as well as the relationship between this degree of deficiency and the severity of the disease [8-10].

The mechanism of VD3 action involves the binding of the activated VD3 receptor/retinoic X receptor (VD3R/ RXR) heterodimeric complex to specific DNA sequences, that controls gene expression [11, 12]. This VD3 action probably alters genes involved in glutamatergic and GABAergic neurotransmissions, calcium regulation, as well as neurotrophic factors and genes involved in neuroprotection [13]. VD3 deficiency may have a negative influence on critical processes necessary for brain function, as neurotransmission, synapse formation, synaptic plasticity, and dendritic arborization [14]. Furthermore, VD3 deficiency does act as a risk factor for PD development [15].

6-Hydroxydopamine (6-OHDA) when injected into the striatum has been shown to cause a retrograde progressive loss of dopaminergic cell bodies in the SNpc, providing a time window that allows for the testing of new therapeutic options [16]. The ability to protect the dopaminergic neurons, mediated by VD3, has already been demonstrated, however the mechanism involved has not been fully elucidated. In addition, studies that provide robustness to the theory of neuroprotective activity of vitamin D still need to be carried out.

In this context, and knowing that VD3 crosses the blood brain barrier [17], the present study was designed to examine, by means of behavioral, neurochemical and immunohistochemical assays, the potential capacity of VD3 to protect nigrostriatal DA neurons against a partial lesion induced by the unilateral striatal injection of 6-OHDA. In addition, the participation of the brain VD3 receptor (VD3R) in this experimental model of PD was also investigated.

\section{Methods}

\section{Drugs and reagents}

Vitamin D (DePura, colecalciferol, VD3) was purchased from Sanofi, São Paulo, SP, Brazil. Vitamin D (VD3) was suspended in an aqueous solution of $1 \%$ DMSO before use. 6-Hydroxydopamine, apomorphine and standards for HPLC measurements were purchased from SigmaAldrich, Saint Louis, MO, USA. Ketamine (Vetanercol) and xylazine (Kensol) were from König Laboratory, Santana de Parnaíba, SP, Brazil. Antibodies were from Merck, NJ, USA, Santa Cruz, CA, USA, or from Abcam, CA, USA. All other reagents were of analytical grade.

\section{Animals}

Male Wistar rats $(250 \mathrm{~g})$ were housed in a 12-h light/ 12-h dark cycle, with water and standard food ad libitum. The project was approved by the Institutional Committee for Animal Experimentation of the Federal University of Ceará, under the no. 107/2015.

\section{Experimental design}

The animals were divided into four experimental groups (ranging from 4 to 10 animals each): Sham operated (SO), non-treated 6-OHDA, and 6-OHDA treated with $1 \mu \mathrm{g} / \mathrm{kg} \mathrm{VD} 3$, for 7 days before surgery (pre-treatment) or for 14 days (post-treatment) after the surgery (6-OHDA+VD3). The SO and the non-treated 6-OHDA groups were treated daily, by gavage with $0.2 \mathrm{~mL}$ distilled water. The SO group was submitted to surgery with the infusion of saline in the right striatum. Fourteen days after surgery, the animals from all groups were submitted to behavioral tests and, shortly thereafter, euthanized for neurochemical and immunohistochemistry studies.

\section{Stereotaxic surgery and 6-OHDA lesion}

For screening new therapeutic alternatives, animal models of Parkinson's disease are widely used. The mostly used animal model of PD consists in the 6-OHDA injection into the rodent striatum. This neurotoxin acts in a retrograde way and is taken up into nigral dopaminergic neurons, initiating cell death by oxidative stress, within a few days [18]. For the experiment, the animals were anesthetized with ketamine $(80 \mathrm{mg} / \mathrm{kg}$, i.p. $)$ and xylazine $(20 \mathrm{mg} / \mathrm{kg}$, i.p.). Before being placed on the stereotaxic apparatus, the animals were submitted to head trichotomy. A Hamilton syringe was used to inject 6-OHDA in two points $(1 \mu \mathrm{L}$ each, corresponding to a total of $12 \mu \mathrm{g}$ 6-OHDA, dissolved in saline, containing $0.2 \%$ ascorbic acid) into the right striatum. For that, a hole was performed with a hand drill. The bregma was the reference point for the following coordinates, which ensure striatal access: first point: AP, +0.5 ; LL, -2.5 ; DV, -5.0 ; second point: AP, -0.5 ; LL, -3.7 ; DV, -6.5 , according to the Paxinos and Watson Atlas (2005). For diffusion of 6-OHDA, the needle was left in place for 5 min before being slowly withdrawn. 


\section{Behavioral tests \\ Open field test}

This test was performed in order to evaluate the exploratory behavior, as an index of spontaneous motor activity. The apparatus $(50 \mathrm{~cm} \times 50 \mathrm{~cm} \times 30 \mathrm{~cm}$, length, width, and height, respectively) was divided into four quadrants of equal size and illuminated by a red light. Each rat was placed in the center of the open field, and the number of crossings was registered for $5 \mathrm{~min}$. After each test, the arena was cleaned with a solution of $70 \%$ alcohol [19].

\section{Rotational behavior induced by apomorphine}

The unilateral injection of 6-OHDA into the striatum triggers motor asymmetry, easily observed after administration of a dopaminergic agonist, such as apomorphine. The motor behavior is characterized by contralateral rotations, in this sensitive test for measuring the degree of striatal lesions [20]. Contralateral rotations for $60 \mathrm{~min}$ were counted after the rats were subcutaneously (s.c.) injected with apomorphine, $1 \mathrm{mg} / \mathrm{kg}$.

\section{Forced swimming test}

This test is based on the observation that when the animals are subjected to a stressful situation, with no possibility for escaping, they adopt a posture of immobility after an initial period of agitation. The animals were placed individually into a cylinder $(40 \mathrm{~cm}$ height and $23 \mathrm{~cm}$ diameter), containing water up to $25 \mathrm{~cm}$ below the top. The immobility time was monitored for $5 \mathrm{~min}$, after an initial $1 \mathrm{~min}$ adaptation period. The reduction of this immobility time is suggestive of an antidepressive-like action. This is a rodent behavioral test used for evaluation of antidepressant efficacy of new compounds and of experiments aimed at rendering or preventing depressive-like states [21, 22].

\section{Neurochemical assays \\ Measurements of striatal dopamine (DA) and DOPAC contents by HPLC}

Degeneration of the dopaminergic nigrostriatal system causes dopaminergic depletion responsible for most of the motor symptoms of Parkinson's disease [23]. Striatal DA and DOPAC levels were measured in 10\% homogenates prepared in $0.1 \mathrm{M} \mathrm{HClO}_{4}$. After sonication for $30 \mathrm{~s}$, the homogenates were centrifuged under refrigeration (15 $\mathrm{min}$ at 20,000 $\mathrm{x} \mathrm{g}$ ), the supernatant was filtered (0.2 $\mu \mathrm{m}$, Millipore), and samples (20 $\mu \mathrm{L}$, each) were injected into a high-performance liquid chromatography (HPLC) column $(\mathrm{C} 18,5 \mu \mathrm{m}, 250 \times 4.6 \mathrm{~mm})$. The mobile phase was $0.163 \mathrm{M}$ citric acid, $\mathrm{pH}$ 3.0, containing $0.02 \mathrm{mM}$ EDTA, with $0.69 \mathrm{mM}$ sodium octanesulfonic acid (SOS), as ion pairing reagent, $4 \% v / v$ acetonitrile and $1.7 \% v / v$ tetrahydrofuran. The concentrations of DA and DOPAC were determined by comparison with standards, and the results were expressed as ng/g tissue.

\section{Determination of striatal nitrite contents}

In this assay, the Griess reagent ( 1 part $0.1 \%$ naphthylethylenediamine dihydrochloride in distilled water plus 1 part $1 \%$ sulfanilamide in $5 \% \mathrm{H}_{3} \mathrm{PO}_{4}$ ) indicates the presence of nitrites in the sample. Striatal homogenates $(10 \%$ in $\mathrm{KCl}$ buffer) were centrifuged (10,000 x g for $10 \mathrm{~min})$, and $100 \mu \mathrm{L}$ supernatants were added to $100 \mu \mathrm{L}$ Griess reagent. This mixture stayed on $\mathrm{RT}$ for $10 \mathrm{~min}$. The standard $\mathrm{NaNO}_{2}$ curve was obtained (in spectrophotometer, at $520 \mathrm{~nm}$ ) and used for calculating the results expressed as micromole nitrite per gram tissue [24].

\section{Determination of striatal lipid peroxidation (tiobarbituric acid reactive substances, TBARS assay)}

Lipid peroxidation expresses oxidative stress induced by ROS reactivity and a largely used method for that is the determination of malondihaldehyde (MDA) in biological samples [25]. The lipid peroxidation products are MDA and 4-hydroxy-2-nonenal (4-HNE), of which MDA is considered a good biomarker of oxidative stress and an end product of lipid peroxidation [26]. Striatal homogenates $(10 \%)$ in $1.15 \% \mathrm{KCl}$ were added $(250 \mu \mathrm{L})$ to $1 \mathrm{~mL}$ $10 \%$ TCA followed by addition of $1 \mathrm{~mL} 0.6 \%$ thiobarbituric acid. After agitation, this mixture was maintained in a water-bath $\left(95-100{ }^{\circ} \mathrm{C}\right)$ for $15 \mathrm{~min}$. Then, the mixture was cooled on ice and centrifuged $(1,500 \times \mathrm{g} / 5$ min). The TBARS content was determined in a plate reader, at $540 \mathrm{~nm}$, with results expressed in micromole MDA per gram tissue. A standard curve with several MDA concentrations was also performed.

Immunohistochemistry assays for tyrosine hydroxylase (TH), dopamine transporter (DAT), VD3 receptor (VD3R), and tumor necrosis factor alpha (TNF-alpha) in rat striata Dopaminergic neurons can be characterized by expression of tyrosine hydroxylase $(\mathrm{TH})$, the rate-limiting enzyme in the catecholamine synthesis pathway [27, 28]. The physiological role of DAT is the re-uptake of released DA into presynaptic DA-terminals. DAT density has been reported to be downregulated in response to dopaminergic lesions, presumably to maintain synaptic dopamine levels [29]. The actions of VD3 are mediated by the vitamin D receptor (VD3R), a ligand-activated transcription factor that functions to control gene expression [11]. Furthermore, evidences indicate the participation of neuroinflammation mediators as TNF-alpha in the pathogenesis of PD [30]. For the immunohistochemical assays, coronal slices of striata were fixed in formaldehyde for $24 \mathrm{~h}$ followed by $70 \%$ alcohol immersion. Striatal sections were cut $(5 \mu \mathrm{m})$, placed on slides, and washed three times with PBS followed by the addition of $3 \%$ hydrogen peroxide in PBS. 
Then, the primary antibodies anti-TH, anti-DAT, anti-VD3R, or anti-TNF-alpha were added after dilution, according to the manufacturers' instructions, in $0.05 \mathrm{M}$ Tris buffer, $\mathrm{pH}$ 7.2-7.6, containing 1\% BSA, and stayed overnight. The next day, the slices were washed two times with PBS followed by the addition of the secondary antibody (yellow reagent or Link, DAKO Cytomation) for $1 \mathrm{~h}$ in a cold chamber. Then, after washing again with PBS, the slices were soaked in streptavidin-peroxidase (red reagent, DAKO Cytomation) for $40 \mathrm{~min}$. After another wash, a DAB solution (3-3-diaminobenzidine tetrahydrochloride, prepared according to the manufacturer's instructions) was applied on the top of the slices, for $30 \mathrm{~s}$, and these were mounted on a free xylol medium, for light microscopy analyses.

\section{Statistical analyses}

All data were expressed as mean $\pm \mathrm{SEM}$. For all parameters, a one-way ANOVA was used followed by the Tukey's post hoc test. The threshold for statistical significance was set to $p<0.05$. All statistical analyses were performed with the GraphPad Prism (Version 6.0). For the immunohistochemical data, the optical density was determined by the Image J software (NIH, USA).

\section{Results}

\section{Behavioral tests}

In the open field test, the non-treated 6-OHDA group showed a reduced motor activity with a $46 \%$ decrease in the number of crossings/5 min. On the contrary, no significant differences were observed in the 6-OHDA group after VD3 treatments, relatively to the SO group. As a whole, treatments with VD3 showed a significant improvement on the animal's mobility (Fig. 1a). In the apomorphine-induced circling behavior, while the non-treated 6-OHDA group showed 252 contralateral rotations/h, compared with that of the SO group, only 21 and 72 contralateral rotations/h were presented by the 6-OHDA groups after VD3 pre- and post-treatments, respectively (Fig. 1b). Importantly, the significant motor asymmetry observed in the non-treated 6-OHDA group, after apomorphine administration, validates the striatal lesion experimental procedure. In the forced swimming test, the non-treated 6-OHDA group showed an almost two times increase in immobility time, compared with the SO group, indicating a depressive-like behavior. Interestingly, the VD3 treated 6-OHDA groups showed values significantly lower than those of the SO group (Fig. 1c).

\section{Neurochemical assays}

\section{Dopamine (DA) and DOPAC determinations in the rat} lesioned striata

A $72 \%$ reduction in DA striatal contents was observed in the non-treated 6-OHDA group, compared with the SO group.
Importantly, no significant alterations were demonstrated in DA levels, in the striata of 6-OHDA groups after VD3 treatments. Additionally, decreases (54\%) were observed in striatal DOPAC contents, in the non-treated 6-OHDA group. Also, DOPAC values in both VD3-treated groups went towards those of the SO groups (Fig. 2).

\section{Nitrite and lipid peroxidation measurements in the rat lesioned striata}

A two times increase in striatal nitrite contents was observed in the non-treated 6-OHDA group, compared with the SO group. On the contrary, no significant changes were seen in the 6-OHDA group, after VD3 treatments, whose values were not different from those of the SO groups. Increases in the striatal lipid peroxidation of almost four times were observed in the non-treated 6-OHDA group, related to the SO group. However, no changes were seen in the 6-OHDA group after pre- or post-VD3 treatments (Fig. 3).

\section{Immunohistochemistry for TH, DAT, TNF-alpha, and VD3R in the rat lesioned striata}

The immunohistochemistry data for $\mathrm{TH}$ revealed an $86 \%$ decrease in the non-treated 6-OHDA groups, compared with the SO groups. Interestingly, no reduction was observed in the 6-OHDA groups, after pre- and post-VD3 treatments (Fig. 4). Similarly, the non-treated 6-OHDA showed an $81 \%$ decrease in DAT immunostaining, relatively to the SO groups. The decreases in DAT values were much lower in the 6-OHDA groups, after pre- and post-VD3 treatments (16 and 22\% decreases, respectively), relatively to the SO groups (Fig. 5). The TNF-alpha data presented a 2.4 times increase in immunostaining for this pro-inflammatory cytokine, in the non-treated 6-OHDA group, in relation to the $\mathrm{SO}$ group. This value dropped to 1.6 and 1.4 times increases after pre- and post-treatments with VD3, respectively (Fig. 6). A significant decrease (77\%) in the striatal VD3R immunoreactivity was demonstrated in the non-treated 6-OHDA group, compared with the SO group. No significant changes were noticed in the striata from both VD3 pre- or post-treatment groups (Fig. 7).

\section{Discussion}

VD3 has been shown to have a close relationship to brain function and evidences for that have significantly increased in the last decades. Although some clinical studies support a role for VD3 neuroprotection in PD [31-35], other studies [36] show no clear causal association between lower VD3 concentration and increased risk for PD. In the present study, we showed that VD3 treatments reversed partially or completely the behavioral, neurochemical, and immunohistochemical alterations, as those observed in the 6-OHDA-induced PD model, in rats. Thus, we observed that VD3 treatments 

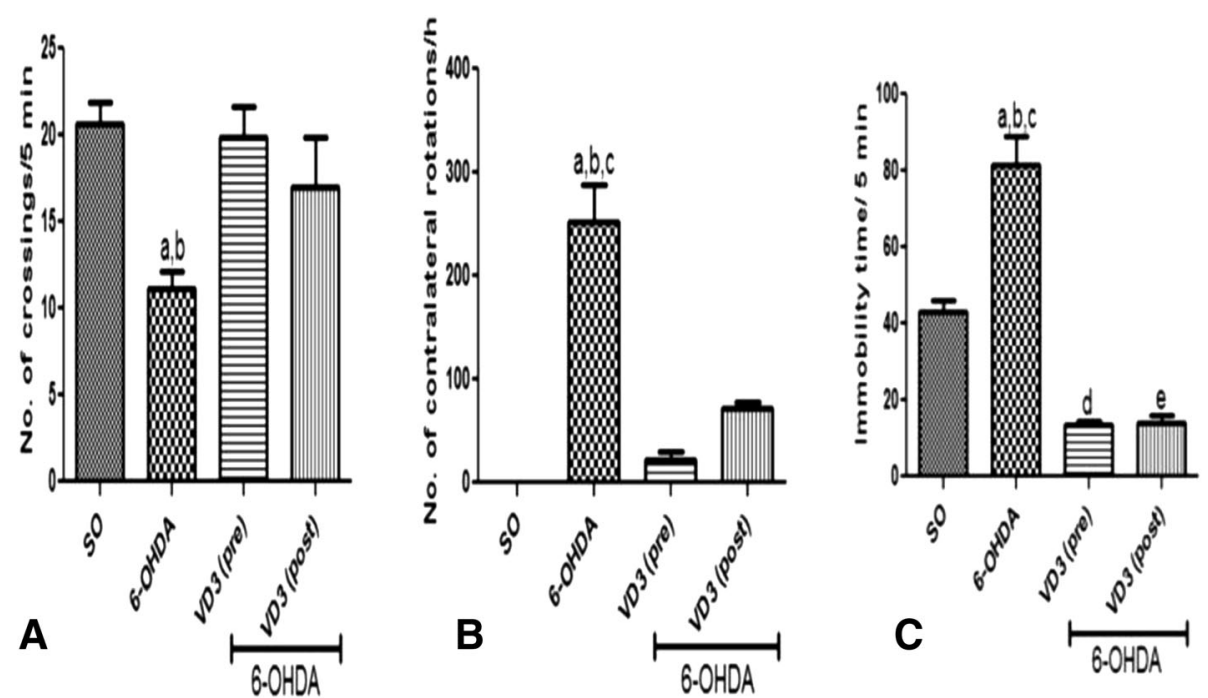

Fig. 1 The pre- and post-treatments with vitamin $D(V D 3,1 \mu \mathrm{g} / \mathrm{kg}$, p.o.) reversed the increased number of crossings (A), the apomorphine-induced circling behavior (B), and the immobility time $(\mathbf{C})$, compared with the non-treated 6-OHDA group. A: a vs. SO, $q=4.608, p<0.05 ; \mathbf{b}$ vs. 6-OHDA+VD3 (pre), $q=4.375, p<0.05$. B: a vs. SO, $q=13.53, p<0.001 ; \mathbf{b}$ vs. $6-\mathrm{OHDA}+\mathrm{VD} 3$ (pre), $q=12.37, p<0.001 ; \mathbf{c}$ vs. OHDA+VD3 (post), $q=9.673, p<0.001 . \mathbf{C}: \mathbf{a}$ vs. SO, $q=8.873, p<0.001 ; \boldsymbol{b}$ vs. $6-\mathrm{OHDA}+\mathrm{VD} 3$ (pre), $q=15.76, p<0.001 ; \mathbf{c}$ vs. $6-\mathrm{OHDA}+\mathrm{VD} 3$ (post), $q=15.63, p<0.001 ; \mathbf{d}$ vs. SO, $q=6.887, p<0.001 ; \mathbf{e}$ vs. SO, $q=6.754, p<0.001$ (one-way ANOVA and Tukey as the post hoc test)

resulted in the attenuation of the apomorphine circling behavior, which correlates positively to the degree of 6-OHDA striatal lesion [20].

Some studies have shown the relationship between VD3 deficiency and motor impairment, associated with a decrease in the number of crossings and rearing in the open field test [37], what corroborates with our results, where VD3 supplementation caused an improvement in the animals' motor coordination. Furthermore, VD3 completely reversed the increased immobility time demonstrated in the non-treated 6-OHDA group, indicating an antidepressive-like behavior. VD3 deficiency has been related with a reduced immobility time, on the forced swimming test [38]. Low VD3 concentrations in the blood seem to be associated with adult depression and postpartum depression [39, 40]. In addition, the correction of VD3
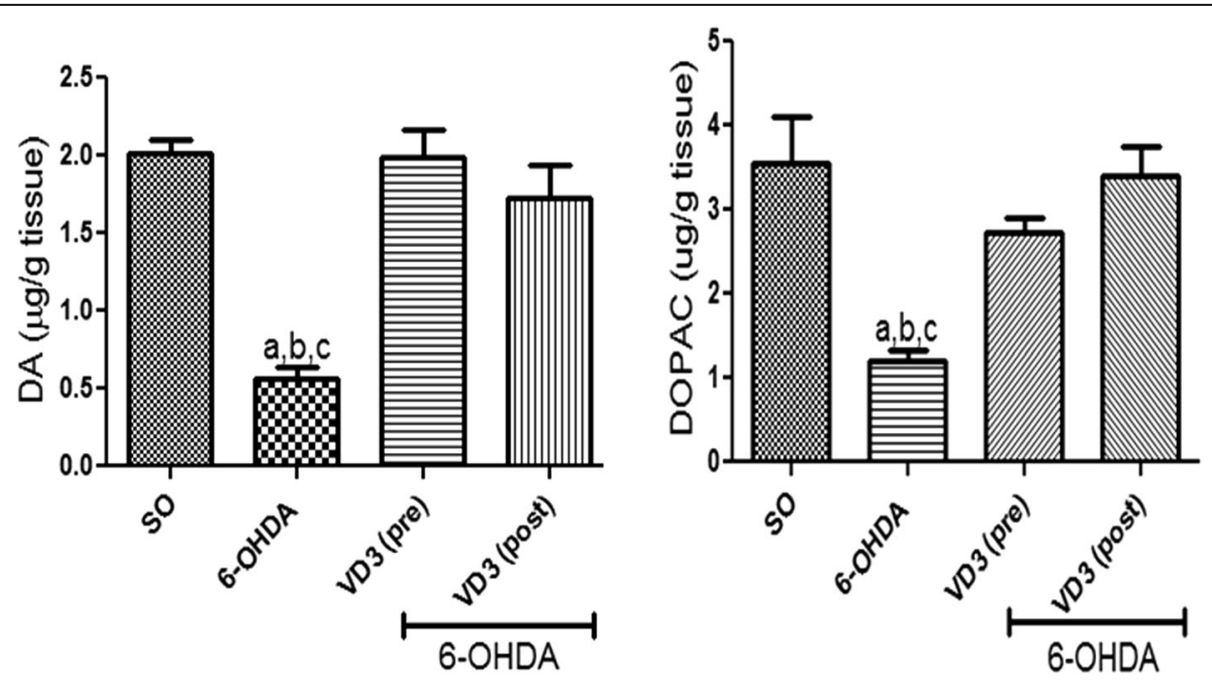

Fig. 2 The pre- and post-treatments with vitamin D (VD3, $1 \mu \mathrm{g} / \mathrm{kg}$, p.o.) reversed DA and DOPAC decreases, demonstrated in the striatal lesioned side of the non-treated 6-OHDA group. DA: a vs. SO, $q=10.16, p<0.001 ; \mathbf{b}$ vs. 6-OHDA+VD3 (pre), $q=10.45, p<0.001$; c vs. $6-\mathrm{OHDA}+\mathrm{VD} 3$ (post), $q=8.166, p<0.001$. DOPAC: a vs. SO, $q=7.290, p<0.001$; b vs. $6-\mathrm{OHDA}+\mathrm{VD} 3$ (pre), $q=5.40, p<0.01 ; \mathbf{c}$ vs. $6-\mathrm{OHDA}+\mathrm{VD3}$ (post), $q=8.072, p<$ 0.001 (one-way ANOVA and Tukey as the post hoc test) 

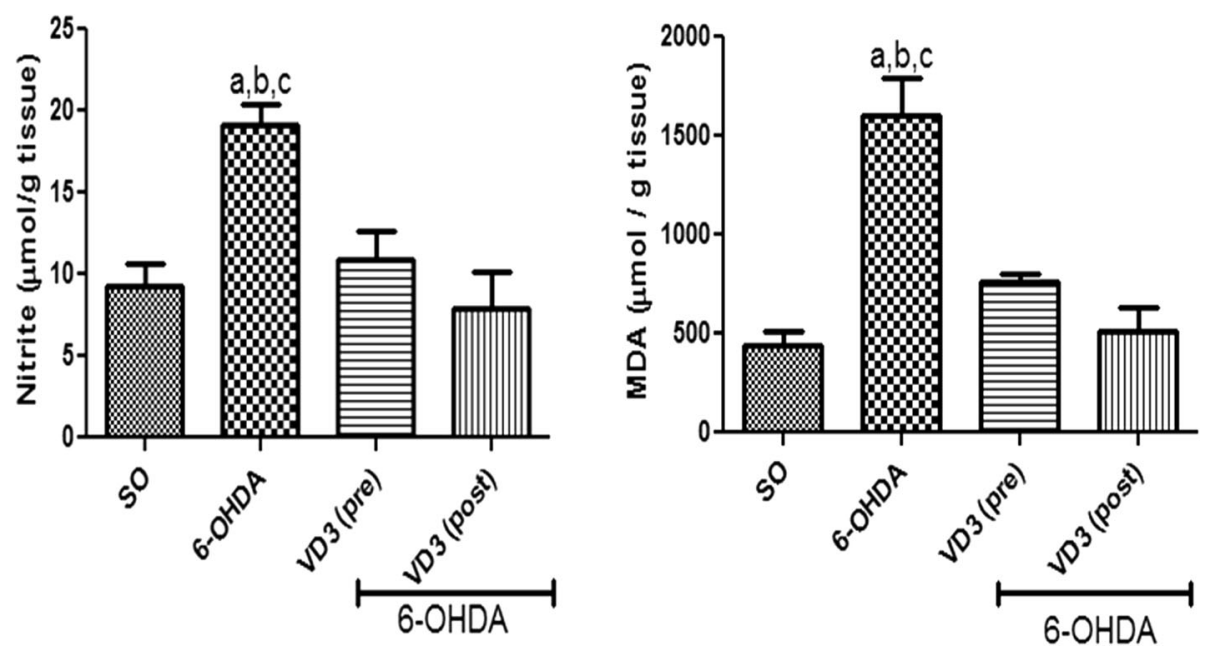

Fig. 3 The pre- and post-treatments with vitamin D (VD3, $1 \mu \mathrm{g} / \mathrm{kg}$, p.o.) reversed the increased nitrite contents and lipid peroxidation (TBARS), observed in the striatal lesioned side of the non-treated 6-OHDA animals. Nitrite: a vs. SO, $q=6.196, p<0.01 ; \mathbf{b}$ vs. $6-\mathrm{OHDA}+\mathrm{VD} 3$ (pre), $q=4.963$, $p<0.05$; c vs. 6-OHDA+VD3 (post), $q=6.120, p<0.01$. TBARS: a vs. SO, $q=11.04, p<0.001 ; \mathbf{b}$ vs. 6-OHDA+VD3 (pre), $q=7.416 ; p<0.001 ; \mathbf{c}$ vs. $6-$ OHDA+VD3 (post), $q=9.040, p<0.001$ (one-way ANOVA and Tukey as the post hoc test)

deficiency or its supplementation may be effective for depression states, as observed in randomized clinical trials $[41,42]$.

VD3 is known to regulate some factors involved with the ontogeny of the dopaminergic system. Thus, neonatal rats whose mothers were deprived of VD3, presented a decreased DA turnover [43]. In addition, VD3 deficiency resulted in altered dopaminergic metabolism in the rat forebrain, with a decreased conversion of
DOPAC to HVA [44]. Evidences indicate that VD3 can not only reduce the extent of dopaminergic denervation, but also offer functional benefit to animals subjected to a Parkinson's disease model [45]. An alteration in the development of the dopaminergic system and on dopamine-mediated behaviors has also been shown in female VD3-deficient rats [44].

A range of studies showed that the substantia nigra presents one of the highest brain concentrations of VD3

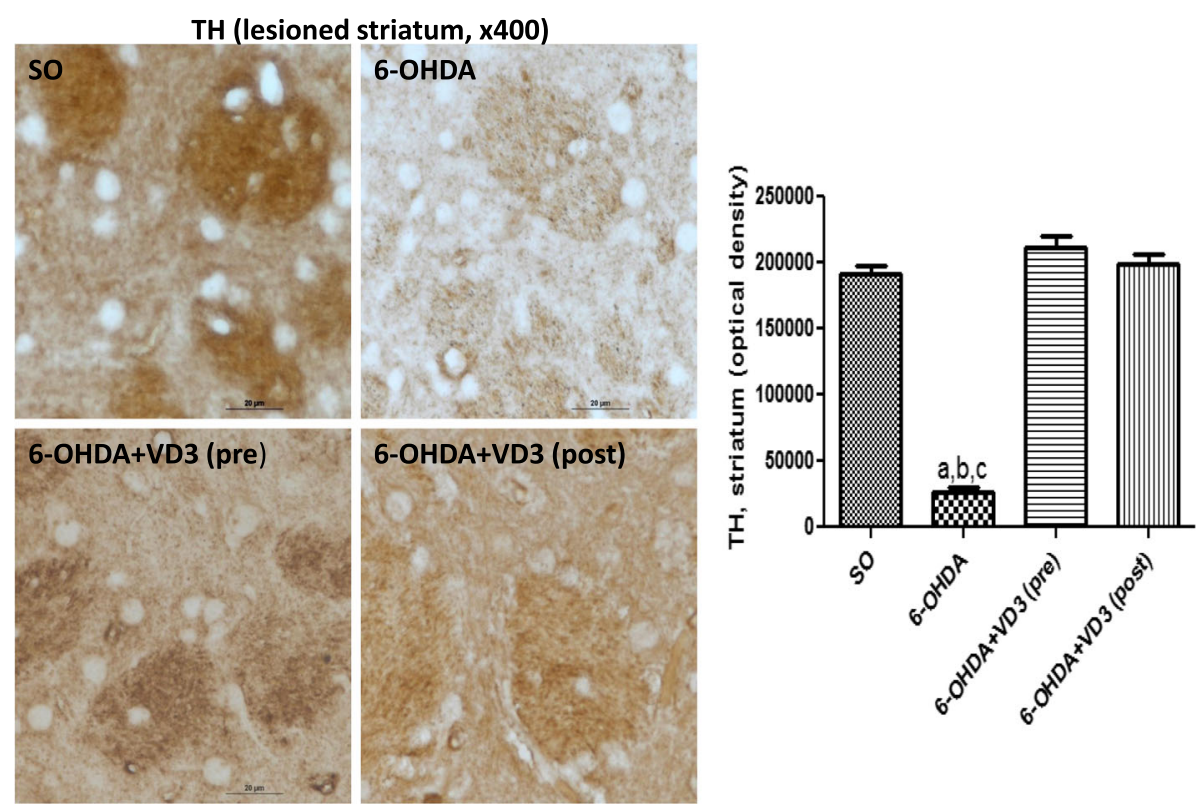

Fig. 4 The pre- and post-treatments with vitamin D (VD3, $1 \mu \mathrm{g} / \mathrm{kg}$, p.o.) reversed the decreased immunostaining for tyrosine hydroxylase (TH), observed in the striatal lesioned side of the 6-OHDA group. a vs. SO, $q=20.80, p<0.001$; b vs. 6-OHDA+VD3 (pre), $q=24.66, p<0.001$; $\mathbf{c}$ vs. 6-OHDA+VD3 (post), $q=22.92, p<0.001$ (one-way ANOVA and Tukey as the post hoc test) 

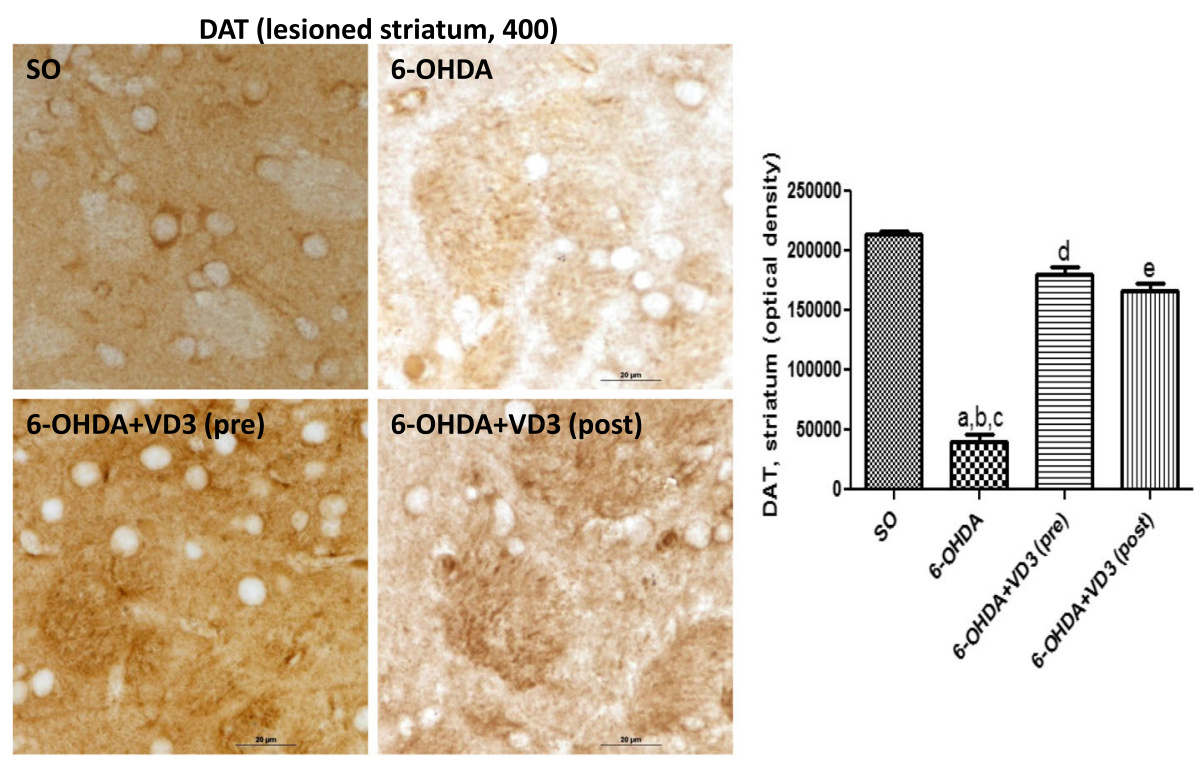

Fig. 5 The pre- and post-treatments with vitamin D (VD3, $1 \mu \mathrm{g} / \mathrm{kg}$, p.o.) partly reversed the decreased immunostaining for the dopamine transporter (DAT), observed in the lesioned striata of the non-treated 6-OHDA rats. a vs. SO, $q=31.29, p<0.001 ; \mathbf{b}$ vs. 6-OHDA+VD3 (pre), $q=25.24, p<0.001$; $\mathbf{c}$ vs. 6-OHDA+VD3 (post), $q=19.81, p<0.001$; $\mathbf{d}$ vs. SO, $q=6.046, p<0.001$; e vs. SO, $q=7.285, p<0.001$ (one-way ANOVA and Tukey as the post hoc test)

receptor (VD3R), indicating the importance of this mediator in dopaminergic pathways [46]. This suggestion was supported by the results obtained on the rotational test, since the animals receiving VD3 did not present motor stereotypy that characterizes the unilateral dopamine depletion. Tyrosine hydroxylase $(\mathrm{TH})$, a rate-limiting enzyme for dopamine synthesis, might be directly modulated by VD3, as observed by immunohistochemistry staining. Previous studies [47] have shown that VD3 administration, before or after the local 6-OHDA injury, partially restores $\mathrm{TH}$ protein and TH-immunoreactive fibers in the striatum and substantia nigra. These effects also increased the glial derived neurotrophic factor (GDNF) protein, contributing to

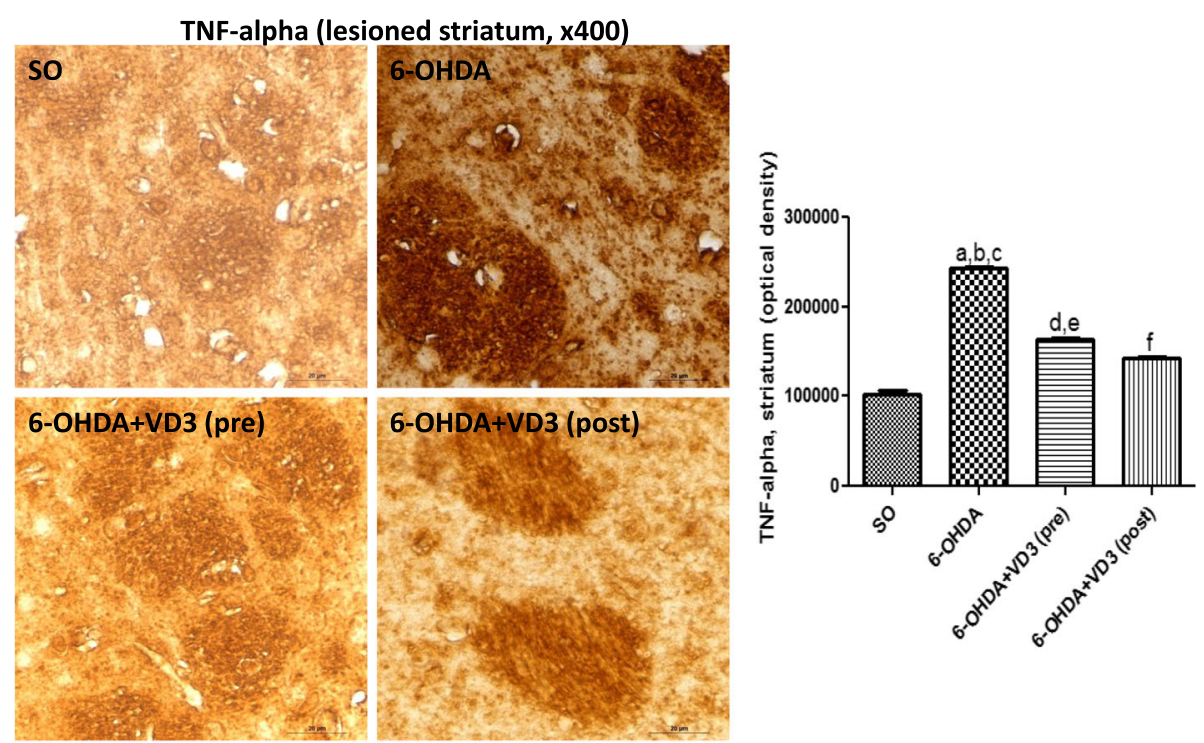

Fig. 6 The pre- and post-treatments with vitamin $D(V D 3,1 \mu \mathrm{g} / \mathrm{kg}$, p.o.) partly reversed the TNF-alpha immunostaining, observed in the lesioned striata of non-treated 6-OHDA animals. a vs. SO, $q=55.10, p<0.001 ; \boldsymbol{b}$ vs. 6-OHDA+ VD3 (pre), $q=31.35, p<0.001$; c vs. 6-OHDA+VD3 (post), $q=39.21, p<0.001$; d vs. SO, $q=23.74, p<0.001$; e vs. $6-\mathrm{OHDA}+\mathrm{VD} 3$ (post), $q=15.89, p<0.001 ; \mathbf{f}$ vs. $6-\mathrm{OHDA}+\mathrm{VD} 3$ (pre), $q=7.857, p<0.001$ (one-way ANOVA and Tukey as the post hoc test) 


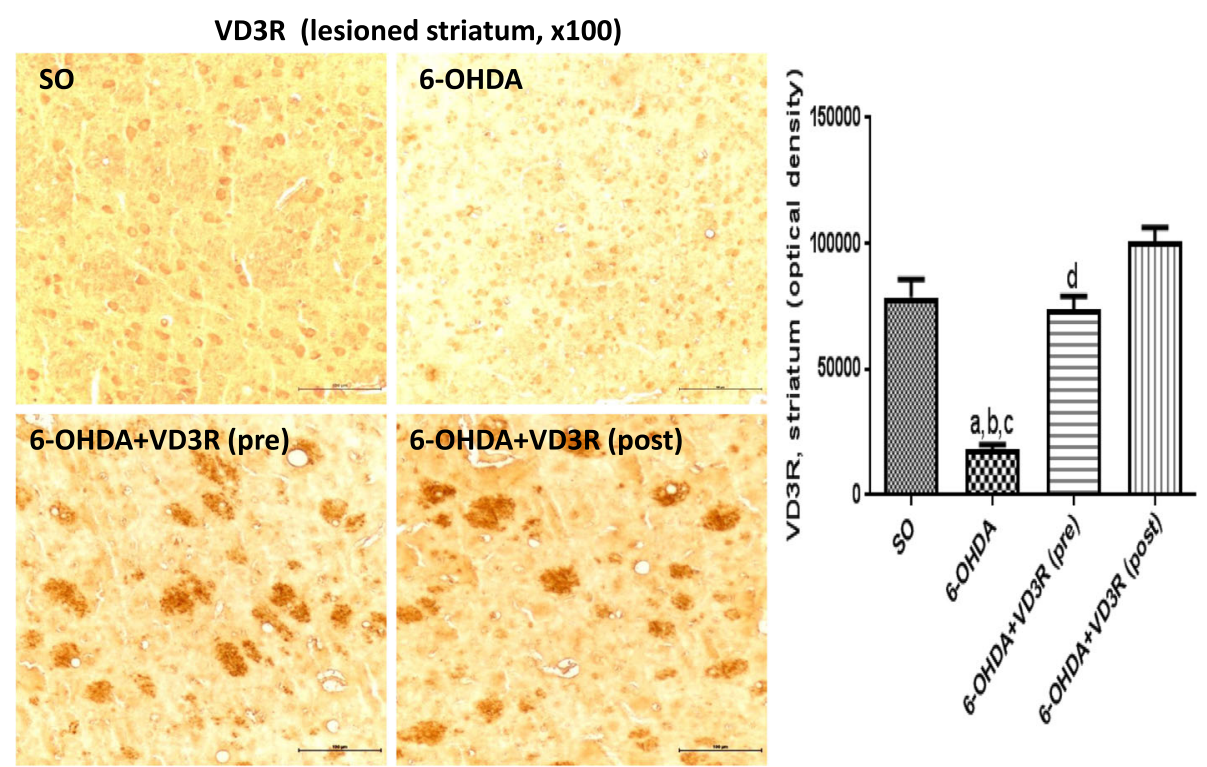

Fig. 7 The pre- and post-treatments with vitamin D (VD3, $1 \mu \mathrm{g} / \mathrm{kg}$, p.o.) partly reversed the VD3 receptor (VD3R) immunostaining, observed in the lesioned striata of the non-treated 6-OHDA animals. a vs. SO, $q=11.38, p<0.001 ; \mathbf{b}$ vs. 6-OHDA+VD3 (pre), $q=10.48, p<0.001 ; \mathbf{c}$ vs. 6-OHDA+VD3 (post), $q=15.49, p<0.001$; d vs. 6-OHDA+VD3 (post), $q=5.009, p<0.05$ (one-way ANOVA and Tukey as the post hoc test)

VD3 neuroprotective effects on dopaminergic neurons. Data from rat mesencephalic cultures of dopaminergic neurons point out to a dose-dependent increase in the number of dopaminergic neurons and upregulation of GDNF, after the addition of VD3 [45].

Several studies have examined the protective effects of VD3 against dopaminergic toxins, as 6-OHDA, showing that VD3 restored partially both nigral and striatal DA levels and also the $\mathrm{TH}$ immunostaining in positive cells [48-50], results that are comparable to ours. The mechanism by which VD3 promotes recovery of dopaminergic release and content remains to be determined, but evidences indicated its potential for affecting calcium metabolism, apoptosis, inflammation, immunomodulation, detoxification, and upregulation of neurotrophins [51-53].

Evidences also suggest that oxidative stress plays a major role in neurodegenerative diseases, including PD $[39,54,55]$. Not only DA metabolism could contribute to that, but also mitochondrial dysfunction, leading to an increase in ROS and activated microglia, and producing NO and ROS during neuroinflammatory responses. The VD3 treatment was shown to increase cell viability and to decrease ROS production, in cone cells under oxidative stress [56]. Furthermore, VD3 deficiency induced ROS production by neutrophil, in spontaneous hypertensive rats [57], and prevented oxidative stress in adipose tissue of diabetic mice [58]. These data agree with ours, showing that VD3 treatments significantly decreased nitrite contents and lipoperoxidation in lesioned striata, as related to the same area of the non-treated
6-OHDA rats, indicating that VD3 presents an antioxidant action.

It is known that, besides the reduction of immunostaining for $\mathrm{TH}$, the reduction of dopaminergic neurons in $\mathrm{PD}$ patients and animal models of parkinsonism leads to substantial loss of the presynaptic markers, such as the dopamine transporter (DAT), probably due to compensatory effects that aim to maintain synaptic functionality in the face of decreased dopamine [59-61]. Nevertheless, in experiments with toxins that induce neuronal death, DAT-deficient immunostaining is a direct reflection of the installed neurodegenerative process $[62,63]$. Thus, DAT staining can be considered an indicator of membrane integrity of dopaminergic neurons.

As expected, in the present work, the striata of the 6-OHDA rats showed a decrease in DAT immunostaining, which was also reversed although to a lower extent. A DAT blockade is observed with some antidepressant drugs [50], and VD3 significantly decreased the immobility time, suggesting an antidepressive-like behavior. Depressive behavior is a common feature of Parkinson's disease patients [64], and clinical studies are consistent with the hypothesis that low VD3 concentration is associated with depression [65]. How VD3 acts to reduce depression is unclear. Evidences indicate that VD3 acts by reducing the increased neuronal levels of $\mathrm{Ca}^{2+}$ associated with depression [66].

All the results presented so far converge to the neuroprotective potential of VD3. Most of its biological actions are now exerted through the nuclear VD3 receptor (VD3R)-mediated control of target genes. VD3R is present 
in mammalian brain and by proteomic techniques was also shown to be present in the adult rodent brain. However, its expression is quantitatively lower in the brain, comparatively with the gut and kidneys from both embryonic and adult tissues [67]. VD3R belongs to the nuclear hormone receptor superfamily, which also includes receptors for cortisol and thyroid hormone, and acts as a ligand-inducible transcription factor $[68,69]$. In the present study, we showed that VD3 treatments increase the immunoreactivity for VD3R, in the striata from non-treated 6-OHDA animals. Furthermore, VD3 treatments activated VD3R expression and attenuated neurological deficits observed in a traumatic brain injury model [70].

VD3 neuroprotective effects involve several independent mechanisms [71] and, among them, the potent immunomodulatory activities in both innate and adaptive immunity [72]. In the last decades, neuroinflammation has been considered an important cause for progression of PD [73, 74]. In the MPTP-induced PD model, researchers demonstrated that the treatment with VD3 attenuates iNOS and pro-inflammatory cytokines expression [75]. The same pattern could be observed in animal models using the 6-OHDA neurotoxin. A post-mortem study showed the increased levels of TNF- $\alpha$ and other inflammatory mediators in the brain of Parkinson's disease patients [76]. VD3 was shown to significantly attenuate the loss of $\mathrm{TH}$-positive neuronal cells, microglial cell activation, iNOS expression, among other typical hallmarks of microglia activation, in an animal model of PD similar to ours [75].

Protein misfolding plays a key role in neurodegenerative diseases and is characterized in PD by the accumulation of intraneuronal aggregates of $\alpha$-synuclein, main component of Lewy bodies. In addition, evidence indicates that $\alpha$-synuclein is a prion-like protein and therefore PD is considered to be a prion-like disease [77-80]. Furthermore, the transcellular propagation of protein aggregation might underlie the progression of neurodegenerative diseases, and $\alpha$-synuclein fibrils are able in PD to spread via cell-to-cell transfer [80, 81]. Most importantly, the cellular form of the prion protein (PrPc) acts as a receptor for $\alpha$-synuclein fibrils [80]. Curiously, Vitamin D2 (ergocalciferol) has been shown to interact with the human PrPc and may be a suitable agent to target PrPc in the brain and, therefore, a potential therapeutic candidate for prion diseases [82].

\section{Conclusion}

Recently (see Additional file 1), we observed significant effects of VD3 (0.5 and $1.0 \mu \mathrm{g} / \mathrm{kg}$, p.o.) after a 7-day treatment in the formalin test of nociception and in the carrageenan-induced paw edema test in mice. We demonstrated that the VD3 treatments inhibited only the second phase of the formalin test, associated with an inflammatory response. In addition, VD3 reduced the edema volume at the $3 \mathrm{rd}$ hour that corresponds to a maximal effect. These data are related to the VD3 inhibition of prostaglandin synthesis (second phase of the formalin test) and to the reduced expression of inflammatory mediators such as COX-2 and iNOS (carrageenan-induced paw edema test). In addition, these results give support to the participation of the anti-inflammatory action of VD3 on its neuroprotective effect.

Therefore, all together, the neuroprotective action of VD3 as demonstrated in the present study, is probably due to its anti-inflammatory and antioxidant properties. VD3 signaling is involved with neurocognitive decline and neurodegenerative diseases. Most importantly, almost all VD3 actions are mediated by the VD3R [83], pointing out to the role of VD3R in the neuroprotective effects of VD3. VD3 has been shown to downregulate the L-type voltage-sensitive calcium channels and to upregulate nerve growth factor. The suppression of VD3R interrupts these events and neurons become more vulnerable to neurodegeneration [84]. Furthermore, VD3, by its anti-inflammatory and antioxidant properties, by its actions on calcium channels and growth factors and, mainly, by increasing the brain VD3R expression, is a strong candidate for submission to well-designed clinical trials, proposing this steroid hormone for prevention or treatment of neurodegenerative diseases as PD.

\section{Additional file}

Additional file 1: Evaluation of the effects of Vitamin D (VD3) on the formalin and carrageenan-induced paw edema tests, after 7-day treatments. (DOCX $131 \mathrm{~kb}$ )

\section{Abbreviations}

6-OHDA: 6-hydroxydopamine; AD: Alzheimer's disease; BSA: Bovine serum albumin; DA: Dopamine; DAB: (3,3'-diaminobenzidine); DAT: Dopamine transporter; DOPAC: 3,4-Dihydroxyphenylacetic acid; GDNF: Glial cell-derived neurotrophic factor; iNOS: Inducible nitric oxide synthase; MDA: Malondialdehyde; PBS: Phosphate buffered saline; PD: Parkinson's disease; ROS: Reactive oxygen species; SNpc: Substantia nigra pars compacta; TBARS: Tiobarbituric acid reactive substances; TH: Tyrosine hydroxylase; TNFalpha: Tumor necrosis factor alpha; VD3: 1,25-dihydroxyvitamin D3;

VD3R: Vitamin D3 receptor; VD3R/RXR: Vitamin D3 nuclear receptor complex with the retinoid $\mathrm{X}$ receptor

\section{Acknowledgements}

The authors thank the technical assistance of Ms. M. Vilani Rodrigues Bastos and to Prof. M.O.L. Viana for the orthographic revision of the manuscript.

\section{Funding}

The authors are grateful to the financial support of the Brazilian National Research Council (CNPq) and to the Coordination for the Improvement of Higher Level Personnel (CAPES).

Authors' contributions

LARL carried out the surgical experiments of the PD model. MJPL helped with the surgical experiments, and animal care and treatments. ROC performed some immunohistochemical assays. FAVL carried out the HPLC 
measurements. KRTN carried out most of the immunohistochemical processing and assays. IBFC was responsible for the experimental design and wrote the manuscript draft. GSBV coordinated and was responsible for the final version of the manuscript. All authors read and approved the final manuscript.

\section{Ethics approval}

All experimental procedures were approved by the Institutional Committee for Animal Experimentation of the Faculty of Medicine of the Federal University of Ceará, Brazil.

\section{Consent for publication}

Not applicable.

\section{Competing interests}

The authors declare that they have no competing interests.

\section{Publisher's Note}

Springer Nature remains neutral with regard to jurisdictional claims in published maps and institutional affiliations.

\section{Author details}

${ }^{1}$ Faculty of Medicine, Federal University of Ceará (UFC), Rua Barbosa de Freitas, 130/1100, Fortaleza, CE 60170-020, Brazil. ²Faculty of Medicine Estácio of Juazeiro do Norte (Estácio/FMJ), Juazeiro do Norte, Brazil. ${ }^{3}$ Federal University of Piauí (UFPI), Picos, Brazil.

\section{Received: 26 March 2018 Accepted: 1 August 2018 Published online: 31 August 2018}

\section{References}

1. Olanow CW, et al. Levodopa in the treatment of Parkinson's disease: current controversies. Mov Disord. 2004;19:997-1005.

2. Didonet JJ, et al. Neuropeptide S counteracts 6-OHDA-induced motor deficits in mice. Behav Brain Res. 2014:266:29-36.

3. Hegarty SV, Sullivan AM, O'Keeffe GW. The epigenome as a therapeutic target for Parkinson's disease. Neural Regen Res. 2016;11:1735-8.

4. Hutson PH, Clark JA, Cross AJ. CNS target identification and validation: avoiding the valley of death or naive optimism? Annu Rev Pharmacol Toxicol. 2017:57:171-87.

5. Kubis AM, Piwowar A. The new insight on the regulatory role of the vitamin D3 in metabolic pathways characteristic for cancerogenesis and neurodegenerative diseases. Ageing Res Rev. 2015;24:126-37.

6. Berridge MJ. Vitamin D cell signalling in health and disease. Biochem Biophys Res Commun. 2015;460:53-71.

7. Berridge MJ. Vitamin D, reactive oxygen species and calcium signaling in ageing and disease. Philos Trans R Soc B. 2016;371:20150434. https://doi.org/10.1098/rstb.2015.0434.

8. Suzuki M, et al. 25-hydroxyvitamin D, vitamin D receptor gene polymorphisms, and severity of Parkinson's disease. Mov Disord. 2012; 27:264-71.

9. Peterson AL, Mancini M, Horak FB. The relationship between balance control and vitamin D in Parkinson's disease-a pilot study. Mov Disord. 2013; 28:1133-7.

10. Suzuki M, et al. Randomized, double-blind, placebo-controlled trial of vitamin D supplementation in Parkinson disease. Am J Clin Nutr. 2013;97: 1004-13.

11. Pike JW, Meyer MB. The vitamin receptor: new paradigm for the regulation of gene expression by 1,25-dihydroxyvitamin D3. Endocrinol Metab Clin N Am. 2010;39:255-69.

12. Christakos S, et al. Vitamin D: metabolism, molecular mechanism of action, and pleiotropic effects. Physiol Rev. 2016;96:365-408

13. Groves NJ, McGrath JJ, Burne TH. Vitamin D as a neurosteroid affecting the developing and adult brain. Annu Rev Nutr. 2014;34:117-41.

14. Groves NJ, Burne TH. Sex-specific attenuated deficits in adult vitamin D deficient BALB/c mice. Physiol Behav. 2016:157:94-101.

15. James LE, Asuni AA. Parkinson's disease and the "sunshine" vitamin. J Alzheimers Dis Parkinsonism. 2013;3:120. https://doi.org/10.4172/2161-0460. 1000120 .
16. Blesa J, Przedboriski S. Parkinson's disease: animal models and dopaminergic cell vulnerability. Front Neuroanat. 2014;8:155. https://doi.org/10.3389/fnana. 2014.00155

17. Gascon-Barre M, Huet PM. Apparent [3H]1,25-dihydroxyvitamin D3 uptake by canine and rodent brain. Am J Phys. 1983;244(3):E266-71.

18. Monnot C, et al. Asymmetric dopaminergic degeneration and levodopa alter functional corticostriatal connectivity bilaterally in experimental parkinsonism. Exp Neurol. 2017;292:11-20.

19. Broadhurst PL. Determinants of emotionality in the rat. I. Situational factors. Br J Psychol. 1957:48:1-12.

20. Francardo $V$, et al. Impact of the lesion procedure on the profiles of motor impairment and molecular responsiveness to L-DOPA in the 6-hydroxydopamine mouse model of Parkinson's disease. Neurobiol Dis. 2011:42:327-40.

21. Petit-Demouliere B, Chenu F, Bourin M. Forced swimming test in mice: a review of antidepressant activity. Psychopharmacology. 2004;177:245-55.

22. Can A, et al. The mouse forced swim test. J Vis Exp. 2012:e3638. https://doi. org/10.3791/3638.

23. Ahlskog JE. Challenging conventional wisdom: the etiologic role of dopamine oxidative stress in Parkinson's disease. Mov Disord. 2005;20:271-82.

24. Green LC, et al. Analysis of nitrate, nitrite, and [15N] nitrate in biological fluids. Anal Biochem. 1982:126:131-8.

25. Draper HH, Hadley M. Malondialdehyde determination as index of lipid peroxidation. Methods Enzymol. 1990;186:421-31.

26. Ayala A, Muñoz MF, Argüelles S. Lipid peroxidation: production, metabolism, and signaling mechanisms of malondialdehyde and 4-hydroxy-2-nonenal. Oxid Med Cell Longevity. 2014;2014:31.

27. Zeiss CJ. Neuroanatomical phenotyping in the mouse: the dopaminergic system. Vet Pathol. 2005;42:753-73.

28. Björklund A, Dunnett SB. Dopamine neuron systems in the brain: an update Trends Neurosci. 2007;30:194-202.

29. Gao R, et al. High frequency electro-acupuncture enhances striatum DAT and D1 receptor expression, but decreases D2 receptor level in 6-OHDA lesioned rats. Behav Brain Res. 2013;237:263-9.

30. More SV, et al. Cellular and molecular mediators of neuroinflammation in the pathogenesis of Parkinson's disease. Mediators Inflamm. 2013; https://doi.org/10.9155/2013/952375.

31. Knekt $P$, et al. Serum vitamin $D$ and the risk of Parkinson's disease. Arch Neurol. 2010;67:808-11.

32. Shen L, Ji HF. Associations between vitamin D status, supplementation, outdoor work and risk of Parkinson's disease: a meta-analysis assessment. Nutrients. 2015;7:4817-27.

33. Shrestha $\mathrm{S}$, et al. Serum 25-hydroxyvitamin D concentrations in midadulthood and Parkinson's disease risk. Mov Disord. 2016;31:972-8.

34. Wang J, et al. Vitamin D and sunlight exposure in newly-diagnosed Parkinson's disease. Nutrients. 2016:8:142. https://doi.org/10.3390/nu8030142.

35. Rimmelzwaan $L M$, et al. Systematic review of the relationship between vitamin D and Parkinson's disease. J Parkinsons Dis. 2016:6:29-37.

36. Larsson SC, et al. International Parkinson's Disease Genomics Consortium (IPDGC). No clear support for a role for vitamin D in Parkinson's disease: a Mendellian randomization study. Mov Disord. 2017;32:1249-52.

37. Altemus $\mathrm{KL}$, et al. Behavioral correlates of vitamin D deficiency. Physio Behav. 1987;39:435-340.

38. Groves NJ, Burne TH. The impact of vitamin D deficiency on neurogenesis in the adult brain. Neural Regen Res. 2017;12(3):393-4.

39. Anglin RES, et al. Vitamin D deficiency and depression in adults: systematic review and meta-analysis. Br J Psychiatry. 2013;202:100-7.

40. Gould JF, et al. Association of cord blood vitamin D at delivery with postpartum depression in Australian women. Aust NZ J Obstet Gynaecol. 2015;55(5):446-52.

41. Mozaffari-Khosravi $\mathrm{H}$, et al. The effect of different single injections of high dose of vitamin D on improving the depression in depressed patients with vitamin D deficiency: a randomized clinical trial. J Clin Psychopharmacol. 2013;33(3):378-85

42. Schaffer JA, et al. Vitamin D supplementation for depressive symptoms: a systematic review and meta-analysis of randomized controlled trials. Psychosom Med. 2014;76(3):190-6.

43. Pertile RA, Cui X, Eyles DW. Vitamin D signaling and the differentiation of developing dopamine systems. Neuroscience. 2016;333:193-203.

44. Kesby JP, Cui X, O'Loan J, JJ MG, Burne TH, Eyles DW. Developmental vitamin D deficiency alters dopamine-mediated behaviors and dopamine transporter function in adult female rats. Psychopharmacology. 2010;208(1):159-68. 
45. Orme RP, et al. The role of vitamin $D_{3}$ in the development and neuroprotection of midbrain dopamine neurons. Vitam Horm. 2016;100: 273-97

46. Eyles DW, et al. Distribution of the vitamin D receptor and 1a-hydroxylase in human brain. J Chem Neuroanat. 2005;29:21-30.

47. Cui $X$, et al. Vitamin D regulates tyrosine hydroxylase expression: N-cadherin a possible mediator. Neuroscience. 2015;304:90-100.

48. Sanchez B, et al. 1,25-Dihydroxyvitamin D3 administration to 6hydroxydopamine-lesioned rats increases glial cell line-derived neurotrophic factor and partially restores tyrosine hydroxylase expression in substantia nigra and striatum. J Neurosci Res. 2009;87:723-32.

49. Kim JS, et al. 1a,25-Dihydroxyvitamin D3 protects dopaminergic neurons in rodent models of Parkinson's disease through inhibition of microglial activation. J Clin Neurol. 2006;2:252-7.

50. Smith MP, et al. Calcitriol protection against dopamine loss induced by intracerebroventricular administration of 6-hydroxydopamine. Neurochem Res. 2006:31:533-9.

51. Wang JY, et al. Vitamin D3 attenuates 6-hydroxydopamine-induced neurotoxicity in rats. Brain Res. 2001;904:67-75.

52. Klotz B, et al. 1,25-Dihydroxyvitamin D3 treatment delays cellular aging in human mesenchymal stem cells while maintaining their multipotent capacity. PLoS One. 2012;7(1):e29959. https://doi.org/10.1371/journal. pone.0029959.

53. Cass WA, et al. Calcitriol promotes augmented dopamine release in the lesioned striatum of 6-hydroxydopamine treated rats. Neurochem Res. 2014; 39:1467-76

54. Blesa J, et al. Oxidative stress and Parkinson's disease. Front Neuroanat. 2015;9:91. https://doi.org/10.3389/fnana.2015.00091.

55. Berridge MJ. Vitamin D and depression: cellular and regulatory mechanisms. Pharmacol Rev. 2017;69:80-92.

56. Tohari AM, Zhou X, Shu X. Protection against oxidative stress by vitamin D in cone cells. Cell Biochem Funct. 2016;34:82-94.

57. Machado CS, et al. Vitamin D3 deficiency increases DNA damage and the oxidative burst of neutrophils in a hypertensive rat model. Mutat Res Genet Toxicol Environ Mutagen. 2016;798:19-26.

58. Manna P, Achari AE, Jain SK. Vitamin D supplementation inhibits oxidative stress and upregulate SIRT1/AMPK/GLUT4 cascade in high glucose-treated 3T3L1 adipocytes and in adipose tissue of high fat diet-fed mice. Arch Biochem Biophys. 2017;615:22-34

59. Gulley JM, Zahniser NR. Rapid regulation of dopamine transporter function by substrates, blockers and presynaptic receptor ligands. Eur J Pharmacol. 2003;479:139-52.

60. Tian $L$, et al. No differential regulation of dopamine transporter (DAT) and vesicular monoamine transporter 2 (VMAT2) binding in a primate model of Parkinson's disease. PLoS One. 2012;7:e31439. https://doi.org/10.1371/ journal.pone.0031439.

61. Afonso-Oramas $\mathrm{D}$, et al. The dopamine transporter is differentially regulated after dopaminergic lesion. Neurobiol Dis. 2010;40:518-30.

62. Chotibut DM, Apple RJ, Michael FS. Dopamine transporter loss in 6-OHDA Parkinson's model is unmet by parallel reduction in dopamine uptake. PLoS One. 2012;7:e52322.

63. Bäck $S$, et al. High correlation between in vivo $\left.\left[{ }^{123}\right]\right] \beta-C I T$ SPECT/CT imaging and post-mortem immunohistochemical findings in the evaluation of lesions induced by 6-OHDA in rats. EJNMMI Res. 2013;3:46. https://doi.org/ 10.1186/2191-219X-3-46.

64. Hwang O. Role of oxidative stress in Parkinson's disease. Exp Neurol. 2013; 22:11-7.

65. Marsh L. Depression and Parkinson's disease: current knowledge. Curr Neurol Neurosci Rep. 2013;13(12):409. https://doi.org/10.1007/s11910-013-0409-5.

66. Dias V, Junn E, Mouradian MM. The role of oxidative stress in Parkinson's disease. J Parkinsons Dis. 2013;3:461-91.

67. Eyles DW, et al. Intracellular distribution of the vitamin D receptor in the brain: comparison with classic target tissues and redistribution with development. Neuroscience. 2014;268:1-9.

68. Kato $\mathrm{S}$. The function of vitamin $\mathrm{D}$ receptor in vitamin $\mathrm{D}$ action. J Biochem. 2000;127:717-22.

69. McGrath JJ, et al. Developmental vitamin D deficiency and risk of schizophrenia: a 10-year update. Schizophr Bull. 2010;36:1073-8.

70. Cui $X$, et al. Vitamin $D$ and the brain: genomic and non-genomic actions. Mol Cell Endocrinol. 2017;453:131-43.
71. Kalueff AV, Eremin KO, Tuohimaa P. Mechanisms of neuroprotective action of vitamin D(3). Biochemist. 2004;69:738-41.

72. Mora JR, Iwata M, Von Andrian UH. Vitamin effects on the immune system: vitamins A and D take centre stage. Nat Rev Immunol. 2008;8:685-98.

73. Tansey MG, Goldberg MS. Neuroinflammation in Parkinson's disease: its role in neuronal death and implications for therapeutic intervention. Neurobiol Dis. 2010;37:510-8.

74. Chen $\mathrm{H}$, et al. Peripheral inflammatory biomarkers and risk of Parkinson's disease. Am J Epidemiol. 2008;167:90-5.

75. Calvello $R$, et al. Vitamin D treatment attenuates neuroinflammation and dopaminergic neurodegeneration in an animal model of Parkinson's disease, shifting M1 to M2 microglia responses. J Neurolmmune Pharmacol. 2017;12:327-39.

76. Mogi M, et al. Tumor necrosis factor-alpha (TNF-alpha) increases both in the brain and in the cerebrospinal fluid from parkinsonian patients. Neurosci Lett. 1994;165:208-10.

77. Olanow CW, Brundin P. Parkinson's disease and alpha synuclein: is Parkinson's disease a prion-like disorder? Mov Disord. 2013;28:31-40.

78. Brabdel JP, et al. Is Parkinson's disease a prion disease? Rev Neurol (Paris). 2015;171:812-24.

79. Wemheuer WM, Wrede A, Schulz-Schaeffer WJ. Types and strains: their essential role in understanding protein aggregation in neurodegenerative diseases. Front Aging Neurosci. 2017;9:187. https://doi.org/10.3389/fnagi.2017.00187.

80. Srivastava KR, Lapidus LJ. Prion protein dynamics before aggregation. Proc Natl Acad Sci U S A. 2017;114:3572-7

81. Stopschinski BE, Diamond MI. The prion model for progression and diversity of neurodegenerative diseases. Lancet Neurol. 2017;16:323-32.

82. Suenaga M, Hiramoto $Y$, Matsunaga $Y$. Vitamin D2 interacts with human PrPc (90-231) and breaks PrPc oligomerization in vitro. Prion. 2013;7:312-8.

83. Ji LL, et al. Changes in the expression of the vitamin D receptor and LVSCC$\mathrm{A} 1 \mathrm{C}$ in the rat hippocampus submitted to prolonged stress. Mol Med Rep. 2004;9(4):1165-70.

84. Gezen-Ak D, Dursun E, Yilmazer S. The effects of vitamin D receptor silencing on the expression of LVSCC-A1C and LVSCC-A1D and the release of NGF in cortical neurons. PLoS ONE. 2011;6(3):e17553. https://doi.org/10.1371/journal.pone.0017553.

\section{Ready to submit your research? Choose BMC and benefit from:}

- fast, convenient online submission

- thorough peer review by experienced researchers in your field

- rapid publication on acceptance

- support for research data, including large and complex data types

- gold Open Access which fosters wider collaboration and increased citations

- maximum visibility for your research: over $100 \mathrm{M}$ website views per year

At BMC, research is always in progress.

Learn more biomedcentral.com/submissions 